\title{
Interfacial tension of the isotropic-nematic interface in suspensions of soft spherocylinders
}

\author{
R. L. C. Vink and T. Schilling \\ Institut für Physik, Johannes Gutenberg-Universität, D-55099 Mainz, Staudinger Weg 7, Germany
}

(Received 18 February 2005; published 31 May 2005)

\begin{abstract}
The isotropic to nematic transition in a system of soft spherocylinders is studied by means of grand canonical Monte Carlo simulations. The probability distribution of the particle density is used to determine the coexistence densities of the isotropic and the nematic phases. The distributions are also used to compute the interfacial tension of the isotropic-nematic interface, including an analysis of finite size effects. Our results confirm that the Onsager limit is not recovered until for very large elongation, exceeding at least $L / D=40$, with $L$ the spherocylinder length and $D$ the diameter. For smaller elongation, we find that the interfacial tension increases with increasing $L / D$, in agreement with theoretical predictions.
\end{abstract}

DOI: 10.1103/PhysRevE.71.051716

PACS number(s): 64.70.Md, 61.20.Ja, 64.75.+g

\section{INTRODUCTION}

On change of density, suspensions of rodlike particles undergo a phase transition between an isotropic fluid phase, where the particle orientations are evenly distributed, and an anisotropic nematic fluid phase, where the particle orientations are, on average, aligned. This phenomenon was explained by Onsager in a theory based on infinitely elongated hard spherocylinders [1]. Onsager theory has been remarkably successful at describing the isotropic to nematic (IN) transition, and still serves as the basis for many theoretical investigations of the properties of liquid crystals. Over the last 20 years, for instance, several groups have investigated the properties of the IN interface using Onsager-type density functional approaches [2-7]. An important finding of these studies is that the interfacial tension $\gamma_{\mathrm{IN}}$ of the IN interface is minimized when the director, which is the axis of average orientation of the particles, lies in the plane of the interface. In the case of in-plane alignment, $\gamma_{\mathrm{IN}}$ is predicted to be very low, but the precise value varies considerably between different authors $[8,9]$. Theoretical estimates for $\gamma_{\mathrm{IN}}$ typically range from 0.156 [7] to 0.34 [3], in units of $k_{\mathrm{B}} T / L D$, with $L$ the rod length, $D$ the rod diameter, $T$ the temperature, and $k_{\mathrm{B}}$ the Boltzmann constant.

Obviously, the Onsager limit of infinite rod length is purely academic. In order to describe more realistic situations, it is necessary to go beyond the Onsager approximation, and consider the case of finite rod length. An example is the theoretical work of Ref. [10], which demonstrates that the interfacial tension in the case of finite rod length is considerably lower than predicted by Onsager theory.

To test the accuracy of the theoretical estimates of $\gamma_{\mathrm{IN}}$, one might envision a direct comparison to experimental data. Unfortunately, this is not straightforward. The models used in theoretical treatments of the IN interface are typically rather simplistic, usually based on a short-ranged pair potential in a system of monodisperse spherocylinders. It is not reasonable to expect quantitative agreement with experiments using these models, because the interactions in the experimental system will be much more complex. For example, polydispersity may be an important factor, and it is not clear to what extent long-range interactions play a role.
Even the experimental determination of the rod dimensions $L$ and $D$, required if a comparison to theory is to be made, presents complications [9].

In order to validate the assumptions made by the various theoretical approaches, it is nevertheless important to test the accuracy of the theoretical predictions. To this end, computer simulations are ideal, because they, in principle, probe the phase behavior of the model system without resorting to approximations. With inexpensive computer power readily available nowadays, several groups have taken the opportunity to investigate the IN transition by means of simulations [11-18]. An example of this approach is Ref. [12], where the coexistence properties of the bulk isotropic and nematic phases of hard spherocylinders are carefully mapped out using Gibbs ensemble Monte Carlo [19]. These simulations generally recover the Onsager limit for long rods, while for shorter rods pronounced deviations show up [12]. Unfortunately, the Gibbs ensemble cannot be used to measure $\gamma_{\mathrm{IN}}$, which is our aim in this work.

To obtain $\gamma_{\mathrm{IN}}$ in simulations, different techniques must be used. One such technique is based on the anisotropy of the pressure tensor. In Ref. [18], this method is applied to suspensions of ellipsoids with axial ratio $\kappa=A / B=15$, where $A$ is the length of the symmetry axis of the ellipsoids, and $B$ that of the transverse axis. The corresponding interfacial tension is $0.006 \pm 0.005 k_{\mathrm{B}} T / B^{2} \approx 0.09 k_{\mathrm{B}} T / A B$ if a hard interaction potential is used, and $0.011 \pm 0.004 k_{\mathrm{B}} T / B^{2}$ $\approx 0.165 k_{\mathrm{B}} T / A B$ using a soft potential. Note that the anisotropy of the pressure tensor is very small, and therefore difficult to measure accurately in practice, as indicated by the error bars.

In Ref. [20], again for (soft) ellipsoids with $\kappa=15$, a value of the interfacial tension $\gamma_{\mathrm{IN}}=0.016 \pm 0.002 k_{\mathrm{B}} T / B^{2}$ $\approx 0.24 k_{\mathrm{B}} T / A B$ is reported. This result was obtained by measuring the capillary broadening of the IN interface. According to capillary wave theory [21], the mean squared amplitudes of the capillary fluctuations are proportional to $1 / \gamma_{\mathrm{IN}}$, and this can be used to obtain the interfacial tension. Unfortunately, capillary wave theory is only valid in the longwavelength limit, such that very large system sizes are required. Moreover, if, as in Ref. [20], periodic boundary conditions are used, two interfaces will be present in the simulation box. Since $\gamma_{\mathrm{IN}}$ is very small, large capillary fluc- 
tuations can occur, and one needs to be aware of interactions between the two interfaces.

Clearly, in order to obtain $\gamma_{\mathrm{IN}}$ more accurately, much more computer power or different simulation techniques are required. Recent advances in grand canonical sampling methods $[22,23]$ have enabled accurate measurements of the interfacial tension in simple fluids [24,25], and complex fluids such as polymer solutions [26] and colloid-polymer mixtures [27]. Our aim in this paper is to apply these techniques to the IN transition in a system of soft spherocylinders, and to extract the corresponding phase diagram and the interfacial tension. Simulations in the grand canonical ensemble offer a number of advantages over the more conventional methods discussed previously. More precisely, in grand canonical simulations, both the coexistence properties can be probed, as in the Gibbs ensemble, as well as the interfacial properties. Additionally, finite-size scaling methods are available that can be used to extrapolate simulation data to the thermodynamic limit [28-31]. It has been demonstrated that grand canonical ensemble simulations combined with novel finite size scaling algorithms can yield results of truly impressive accuracy [31].

This article is structured as follows: First, we introduce the soft spherocylinder model used in this work. Next, we describe the grand canonical Monte Carlo method, and explain how the coexistence properties and the interfacial tension are obtained. Finally, we present our results, followed in the last section by a discussion and an outlook to future work.

\section{MODEL}

In this study, the particles are modeled as repulsive soft spherocylinders of elongation $L$ and diameter $D$. Hard spherocylinders can, in principle, be dealt with, too, but they severely decrease the efficiency of the simulation (the acceptance rates in simulations of hard spherocylinders are typically 1000 times lower as compared to the soft spherocylinders considered in this work [32]). The interaction between two rods $A$ and $B$ is given by a pair potential of the form

$$
V_{A B}(r)= \begin{cases}\epsilon, & r<D, \\ 0, & \text { otherwise }\end{cases}
$$

with $r$ the distance between two line segments of length $L$; see Fig. 1. The total energy is thus proportional to the number of overlaps in the system. In this work, the rod diameter $D$ is taken as unit of length, and $k_{\mathrm{B}} T$ as a unit of energy. The strength of the potential is set to $\epsilon=2$. Note that in the limit $\epsilon \rightarrow \infty$, this model approaches a system of infinitely hard rods.

To study the IN transition, we typically use the density and the rod alignment as order parameters. Note that both the isotropic and the nematic phase are fluid phases, in the sense that long-range positional order of the centers of mass is absent. In the nematic phase, however, there is orientational order where, on average, the rods point in one direction (called the director). In the isotropic phase, on the other hand, there is no orientational order. Since the density of the nematic phase is slightly higher than of the isotropic phase,

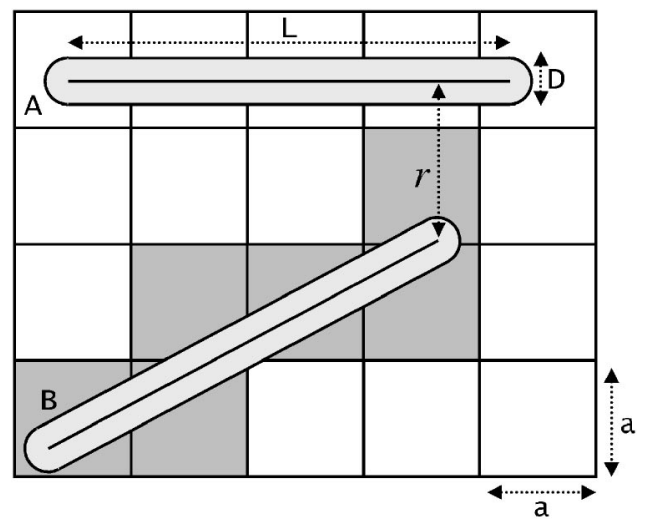

FIG. 1. Two-dimensional representation of the simulation model of this work. The liquid crystals are modeled as soft spherocylinders with elongation $L$ and diameter $D$. Two rods A and B interact via the pair potential of Eq. (1), which is a function of their minimum distance $r$ only. If the rods overlap, the system pays a constant energy cost $\epsilon$. To speed up the determination of overlap, the simulation box is subdivided into cubic cells with edge length $a$; see the details in text.

we may use the particle number density $\rho=N / V$ to distinguish between both phases, with $N$ the number of rods in the system and $V$ the volume of the simulation box. Following convention, we also introduce the reduced density $\rho^{\star}$ $=\rho / \rho_{\mathrm{cp}}$, with $\rho_{\mathrm{cp}}=2 /(\sqrt{2}+(L / D) \sqrt{3})$ the density of regular close packing of hard spherocylinders. Orientational order is as usual measured by the $S_{2}$ order parameter, defined as the maximum eigenvalue of the orientational tensor $Q$ :

$$
Q_{\alpha \beta}=\frac{1}{2 N} \sum_{i=1}^{N}\left(3 u_{i \alpha} u_{i \beta}-\delta_{\alpha \beta}\right) .
$$

Here, $u_{i \alpha}$ is the $\alpha$ component $(\alpha=x, y, z)$ of the orientation vector $\vec{u}_{i}$ of rod $i$ (normalized to unity), and $\delta_{\alpha \beta}$ is the Kronecker delta. In the case of orientational order, such as in the nematic phase, $S_{2}$ assumes a value close to one, while in the disordered isotropic phase, $S_{2}$ is close to zero.

\section{SIMULATION METHOD}

The simulations are performed in the grand canonical ensemble. In this ensemble, the volume $V$, the temperature $T$, and the chemical potential $\mu$ of the rods are fixed, while the number of rods $N$ inside the simulation box fluctuates. The insertion and removal of rods are attempted with equal probability, and accepted with the standard grand canonical Metropolis rules, given by $A(N \rightarrow N+1)=\min [1,[V /(N$ $\left.+1)] e^{-\beta \Delta E+\beta \mu}\right]$ and $A(N \rightarrow N-1)=\min \left[1,(N / V) e^{-\beta \Delta E-\beta \mu}\right]$, with $\Delta E$ the energy difference between the initial and final state, and $\beta=1 / k_{\mathrm{B}} T[29,33]$. The simulations are performed in a three-dimensional box of size $L_{x} \times L_{y} \times L_{z}$ using periodic boundary conditions in all directions. In this work, we fix $L_{x}=L_{y}$, but we allow for elongation in the remaining direction $L_{z} \geqslant L_{x}$. Moreover, to avoid double interactions between rods through the periodic boundaries, we set $L_{x}>2 L$.

During the simulations, we measure the probability $P(N)$, defined as the probability of observing a system containing $N$ 


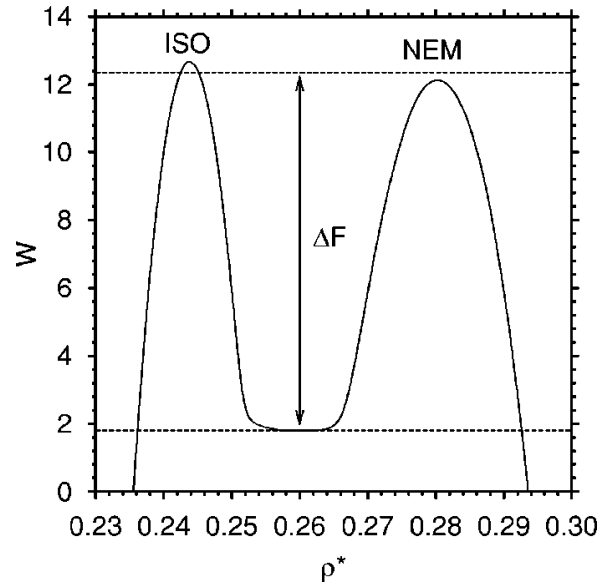

FIG. 2. Coexistence distribution $W=k_{B} T \ln P(N)$ of the isotropic to nematic transition in a system of soft rods interacting via Eq. (1) with $\epsilon=2$ and $L / D=15$. The low-density peak corresponds to the isotropic phase (ISO), the high-density peak to the nematic phase (NEM), and the barrier $\Delta F$ to the free energy difference between the two phases $(\Delta F$ is given by the average peak height as measured from the minimum in between the peaks). The above distribution was obtained using box dimensions $L_{x}=2.1 L$ and $L_{z}=8.4 L$. The coexistence value of the chemical potential reads as $\mu=5.15$ and was obtained using the equal area criterion described in the text.

rods. Note that the shape of the distribution will depend on the rod elongation $L / D$, the temperature $T$, and the chemical potential $\mu$. Moreover, there may be finite-size effects, introducing additional dependences on the box dimensions $L_{x}$ and $L_{z}$. At phase coexistence, the distribution $P(N)$ becomes bimodal, with two peaks of equal area; one located at small values of $N$ corresponding to the isotropic phase, and one located at high values of $N$ corresponding to the nematic phase. A typical coexistence distribution is shown in Fig. 2, where the logarithm of $P(N)$ is plotted. Coexistence is determined using the equal area rule [34]. At coexistence, the equal area rule implies that $\int_{0}^{\langle N\rangle} P(N) d N=\int_{\langle N\rangle}^{\infty} P(N) d N$, with $\langle N\rangle$ the average of the full distribution $\langle N\rangle=\int_{0}^{\infty} N P(N) d N$, where we assume that $P(N)$ has been normalized to unity, $\int_{0}^{\infty} P(N) d N=1$. The coexistence density of the isotropic phase follows trivially from the average of $P(N)$ in first peak $\rho_{\text {ISO }}=(2 / V) \int_{0}^{\langle N\rangle} N P(N) d N$, and similarly for the nematic phase $\rho_{\mathrm{NEM}}=(2 / V) \int_{\langle N\rangle}^{\infty} N P(N) d N$, where the factors of 2 are a consequence of the normalization of $P(N)$.

The interfacial tension $\gamma_{\mathrm{IN}}$ is extracted from the logarithm of the probability distribution $W \equiv k_{\mathrm{B}} T \ln P(N)$. Since $-W$ corresponds to the free energy of the system, the average height $\Delta F$ of the peaks in $W$, measured with respect to the minimum in between the peaks, equals the free energy barrier separating the isotropic from the nematic phase. When the overall density of the system is in the interval between the peaks $\rho_{\text {ISO }} \ll \rho \ll \rho_{\text {NEM }}$, coexistence between an isotropic and nematic domain is observed. A snapshot of the system in this regime reveals a slab geometry, with one isotropic region, and one nematic region, separated by an interface (because of periodic boundary conditions, there are actually two interfaces). An example snapshot is shown in Fig. 3. Note

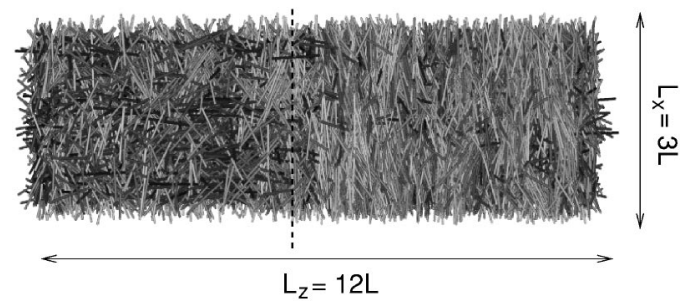

FIG. 3. Snapshot of a system of soft spherocylinders at IN coexistence. The spherocylinders are shaded according to their orientation. On the left side of the dashed line the system is isotropic, on the right side it is nematic. The second interface coincides with the boundaries of the box in the elongated direction.

that the director of the nematic phase lies in the plane of the interfaces. This was the typical case for the snapshots studied by us, and is consistent with the theoretical prediction that in-plane alignment yields the lowest free energy.

The barrier $\Delta F$ in Fig. 2 thus corresponds to the free energy cost of having two interfaces in the system. Since, in this work, the box dimensions are chosen such that $L_{x}=L_{y}$ and $L_{z} \geqslant L_{x}$, the interfaces will be oriented perpendicular to the elongated direction, since this minimizes the interfacial area, and hence the free energy of the system. The total interfacial area in the system thus equals $2 L_{x}^{2}$. Since the interfacial tension is simply the excess free energy per unit area, we may write

$$
\gamma_{\mathrm{IN}}\left(L_{x}\right)=\Delta F /\left(2 L_{\mathrm{x}}^{2}\right)
$$

with $\gamma_{\mathrm{IN}}\left(L_{x}\right)$ the interfacial tension in a finite simulation box with lateral dimension $L_{x}$ [28]. To obtain the interfacial tension in the thermodynamic limit, one can perform a finite size scaling analysis [28] to estimate $\lim _{L_{\mathrm{x}} \rightarrow \infty} \gamma_{\mathrm{IN}}\left(L_{\mathrm{x}}\right)$. Alternatively, away from any critical point, the most dominant finite size effects will likely stem from interactions between the two interfaces. In this case, it is feasible to use an elongated simulation box with $L_{z} \gg L_{x}$, such as in Fig. 3. The advantage of using an elongated simulation box is that interactions between the interfaces are suppressed. This enhances a flat region in $W$ between the peaks, indicating that the interfaces are no longer interacting, and that finite size effects will likely be small. In this work, both approaches will be used.

If the free energy barrier $\Delta F$ is large, transitions between the isotropic and the nematic phase become less likely, and the simulation will spend most of the time in only one of the two phases. A crucial ingredient in our simulation is therefore the use of a biased sampling technique. We use successive umbrella sampling [23] to enable accurate sampling in regions where $P(N)$, due to the free energy barrier separating the phases, is very small. Note also that phase coexistence is only observed if the chemical potential $\mu$ is set equal to its coexistence value. This value is in general not known at the start of the simulation, but it may easily be obtained by using the equation $P\left(N \mid \mu_{1}\right)=P\left(N \mid \mu_{0}\right) e^{\beta\left(\mu_{1}-\mu_{0}\right) N}$, with $P\left(N \mid \mu_{\alpha}\right)$ the probability distribution $P(N)$ at chemical potential $\mu_{\alpha}$. In the simulations, we typically set the chemical potential to zero and use successive umbrella sampling to obtain the corre- 
sponding probability distribution. We then use the above equation to obtain the desired coexistence distribution, in which the area under both peaks is equal.

\section{NUMERICAL OPTIMIZATIONS}

Most of the CPU time in our simulations is spent on calculating the distance $r$ between two line segments; see Fig. 1 . Naturally, one tries to minimize the number of calls to the routine that determines the distance. To this end, we use a cubic linked cell structure, which is schematically illustrated in Fig. 1. The crucial point is that the lattice constant $a$ is chosen such that $D<a<L$. To determine if rod B in Fig. 1 overlaps with any of the other rods in the system, it is sufficient to consider only those rods contained in the cubes intersected by rod B (shaded gray), plus the rods contained in the nearest and next-nearest neighbors of these cubes. Since the isotropic to nematic transition occurs at low density, most cubes will be empty, resulting in a substantial efficiency gain. Some CPU time is used for manipulating the linked cell structure, but for large systems $(\approx N>1500)$ and long rods $(\approx L / D>10)$, the gain in efficiency is already a factor of 5 . Some fine tuning is required to obtain an optimal value of the lattice constant. We found that $a \approx 0.2 L$ typically gives good results.

A further optimization concerns the calculation of the $S_{2}$ order parameter; see Eq. (2). In a naive implementation, determining the orientational tensor $Q$ involves an $\mathcal{O}(N)$ loop over all rods in the system. In our implementation, the tensor elements of $Q$ are updated after each accepted Monte Carlo move, which can be done at the cost of only a few additions and multiplications. Since we keep the tensor elements updated throughout the simulation, the $\mathcal{O}(N)$ loop of Eq. (2) never needs to be carried out. Finally, to determine the maximum eigenvalue of $Q$, we do not use a numerical scheme, but instead use the exact expression for the roots of a third degree polynomial. The advantage of this implementation is that the value of $S_{2}$ is known exactly throughout the simulation, at a cost exceeding no more than one percent of the total invested CPU time.

We conclude this section with a few benchmarks. For $\epsilon$ $=2$ in Eq. (1), we found that the acceptance rate of grand canonical insertion is around 9\% in the isotropic phase, and it decreases to around $6 \%$ in the nematic phase. The acceptance rates are rather insensitive to $L / D$. With the optimized implementation described in this section, we can typically generate 5000-8000 accepted grand canonical moves per second on a $2.2 \mathrm{GHz}$ AMD Opteron processor.

\section{RESULTS}

\section{A. Phase diagram}

We first use our grand canonical Monte Carlo scheme to determine the IN phase diagram of the soft spherocylinder system of Eq. (1) using $\epsilon=2$. For several rod elongations $L / D$, we measured the distribution $P(N)$, from which $\rho_{\text {ISO }}$ and $\rho_{\text {NEM }}$ were obtained. The system size used in these simulations is typically $L_{x}=L_{y}=2.1 L$ and $L_{z}=4.2 L$. In Fig. 4 , we plot the reduced density of the isotropic and the nematic

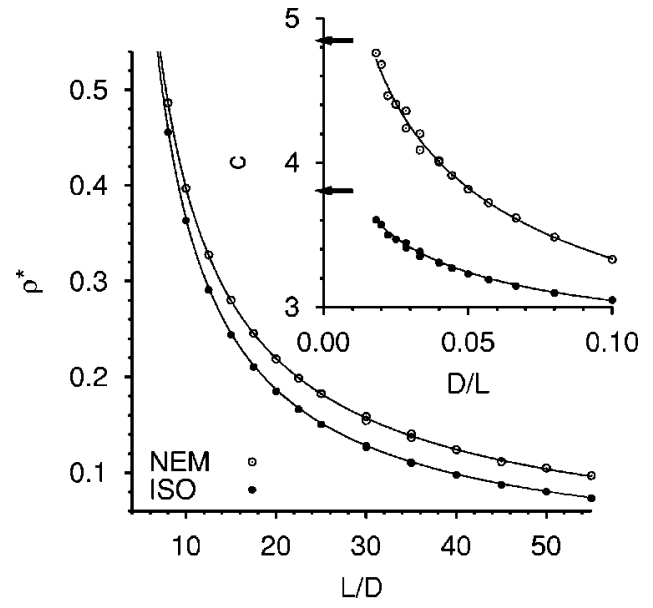

FIG. 4. Soft spherocylinder phase diagram of the IN transition using $\epsilon=2$ in Eq. (1). Shown is the reduced density $\rho^{\star}$ of the isotropic phase (closed circles) and of the nematic phase (open circles) as a function of $L / D$. The inset shows the concentration variable $c$ as a function of $D / L$ for both the isotropic and the nematic phase. The lower and upper arrow in the inset mark the Onsager limit $D / L \rightarrow 0$ for the isotropic and the nematic phase, respectively. The lines connecting the points serve as a guide to the eye.

phase as function of $L / D$. We observe that the phase diagram is qualitatively similar to that of hard spherocylinders [12], the quantitative difference being that, for soft rods, the IN transition is shifted toward higher density. The inset of Fig. 4 shows the concentration variable $c=\pi D L^{2} \rho / 4$ as a function of $D / L$. For hard spherocylinders, Onsager theory predicts that $c_{\mathrm{ISO}}=3.29$ and $c_{\mathrm{NEM}}=4.19$ in the limit of an infinite rod length, or equivalently $D / L \rightarrow 0$. In case of the soft potential of Eq. (1), these values must be multiplied by $\left(1-e^{-\beta \epsilon}\right)^{-1}$ $\approx 1.16$ for $\epsilon=2$. In the inset of Fig. 4, the corresponding limits are marked with arrows. As in Ref. [12], we observe that the simulation data for the isotropic phase smoothly approach the Onsager limit, while the nematic branch of the binodal seems to overshoot the Onsager limit. This we attribute to equilibration problems. To simulate the IN transition in the limit $D / L \rightarrow 0$, large system sizes are required, and it becomes increasingly difficult to obtain accurate results. To quantify the uncertainty in our measurements, additional independent simulations for rod elongation $L / D=25$, 30 , and 35 were performed. The corresponding data are also shown in Fig. 4. For $L / D \geqslant 30$, we observe significant scatter, while for $L / D \leqslant 25$, the uncertainty is typically smaller than the symbol size used in the plots.

\section{B. Interfacial tension}

Next, the interfacial tension $\gamma_{\text {IN }}$ of the IN interface is determined for $L / D=10$ and $L / D=15$. Unfortunately, the system size used to compute the phase diagram in the previous section was insufficient to accurately extract the interfacial tension because no flat region between the peaks in $P(N)$ could be distinguished. This indicates that the interfaces are still strongly interacting. To properly extract the interfacial tension, much larger systems turned out to be required. In this case, care must be taken in the sampling procedure. 

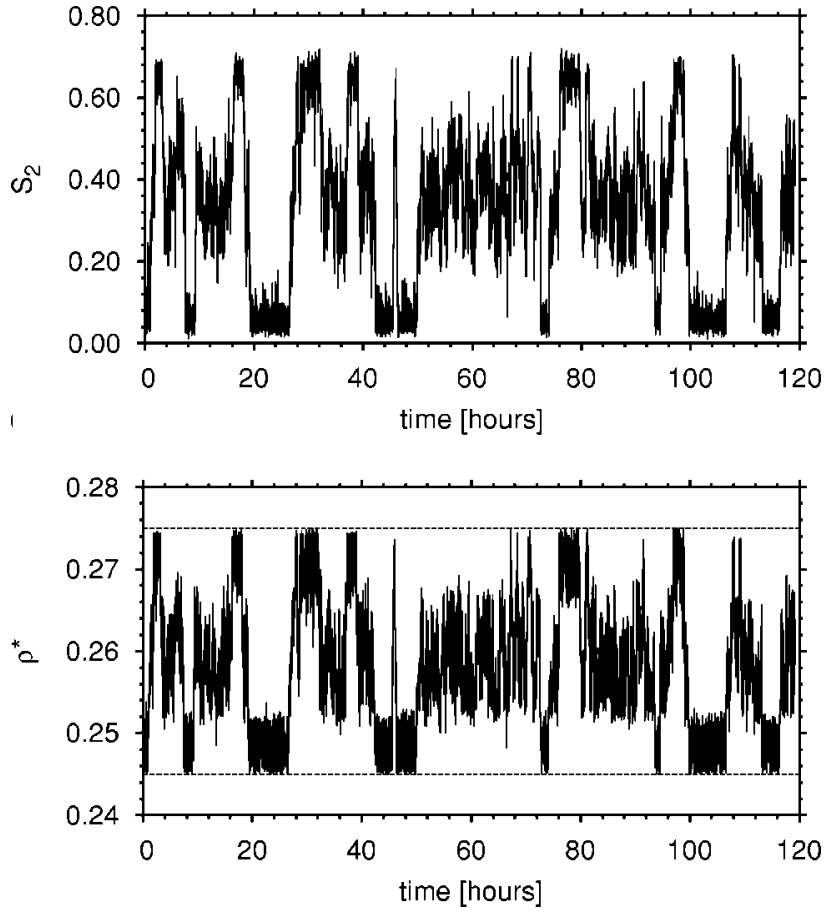

FIG. 5. Monte Carlo time series of a biased grand canonical simulation. The top frame shows the $S_{2}$ order parameter as a function of the invested CPU time, the lower frame the reduced density, with CPU time expressed in hours on a $2.6 \mathrm{GHz}$ Pentium. During the simulation, the reduced density was confined to the interval $0.245<\rho^{\star}<0.275$, as indicated by the horizontal lines in the lower figure. The data were obtained using $L / D=15, \epsilon=2, L_{x}=2.1 L$ and $L_{z}=8.4 L$, which are the same parameters as used in Fig. 2.

Many sampling schemes, especially the ones that are easy to implement, such as successive umbrella sampling, put a bias on the density only. Such schemes tend to "get stuck" in metastable droplet states when the system size becomes large [26]. As a result, one may have difficulty reaching the state with two parallel interfaces, in which case Eq. (3) cannot be used.

Therefore, for large systems, one must carefully check the validity of the simulation results. To this end, we occasionally inspect simulation snapshots. For sufficiently elongated simulation boxes $L_{z} \gg L_{x}$ and at densities inside the coexistence region $\rho_{\text {ISO }} \ll \rho \ll \rho_{\text {NEM }}$, we indeed observe two planar interfaces oriented perpendicular to the elongated direction, in accord with Fig. 3. To further check the consistency of the measured distributions $P(N)$, we performed a number of additional grand canonical simulations using a biased Hamiltonian of the form $\mathcal{H}=\mathcal{H}_{0}+W$, with $\mathcal{H}_{0}$ the Hamiltonian of the real system defined by Eq. (1) and $W=-k_{\mathrm{B}} T \ln P(N)$. If the measured $P(N)$ is indeed the equilibrium coexistence distribution of the real system, a simulation using the biased Hamiltonian should visit the isotropic and the nematic phase equally often on average $[23,35]$. This is illustrated in the top frame of Fig. 5, which shows the $S_{2}$ order parameter as a function of the elapsed simulation time during one such biased simulation. Indeed, we observe frequent transitions between the isotropic $\left(S_{2} \sim 0\right)$ and the nematic phase $\left(S_{2} \sim 1\right)$. Also shown in Fig. 5 is the corresponding time series of the

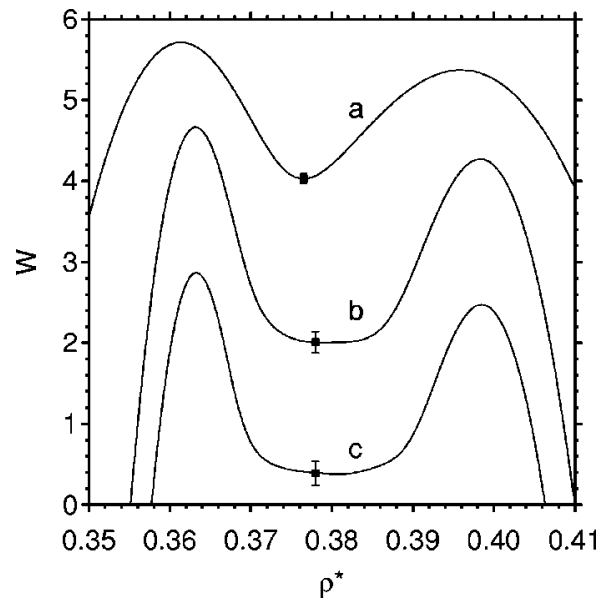

FIG. 6. Coexistence distributions $W=k_{\mathrm{B}} T \ln P(N)$ of soft spherocylinders with $L / D=10$ and $\epsilon=2$ for various system sizes. In each of the above distributions, the lateral box dimension was fixed at $L_{x}=L_{y}=2.3 L$, while the perpendicular dimension was varied: (a) $L_{z}=2.3 L$; (b) $L_{z}=10.35 L$; (c) $L_{z}=13.8 L$. The corresponding free energy barriers $\Delta F$ are (a) $1.52 \pm 0.05$; (b) $2.47 \pm 0.13$; (c) $2.29 \pm 0.15$, in units of $k_{\mathrm{B}} T$. The error bars indicate the magnitude of the scatter in $\Delta F$ for a number of independent measurements.

reduced density. In case a perfect estimate for $P(N)$ could be provided, the measured distribution in the biased simulation will become flat in the limit of a long simulation time. The deviation from a flat distribution can be used to estimate the error in $P(N)$, or alternatively, to construct a better estimate for $P(N)$. The latter approach was in fact adopted by us. First, successive umbrella sampling is used to obtain an initial estimate for $P(N)$. This estimate is then used as the input for a number of biased simulations using the modified Hamiltonian, and improved iteratively each time.

To obtain the interfacial tension, the most straightforward approach is to fix the lateral box dimensions at $L_{x}=L_{y}$, and to increase the elongated dimension $L_{z} \gg L_{x}$ until a flat region between the peaks in the distribution $P(N)$ appears. For soft spherocylinders of elongation $L / D=10$, the results of this procedure are shown in Fig. 6. Indeed, we observe that the region between the peaks becomes flatter as the elongation of the simulation box is increased. Unfortunately, even for the largest system that we could handle, the region between the peaks still displays some curvature. In other words, the interfaces are still interacting, indicating that even more extreme box elongations are required. Ignoring this effect, and applying Eq. (3) to the largest system of Fig. 6, we obtain for the interfacial tension $\gamma_{\mathrm{IN}}=0.0022 k_{\mathrm{B}} T / D^{2}$. For rod elongation $L / D=15$, the distribution of the largest system that we could handle is shown in Fig. 2. The height of the barrier reads as $\Delta F=10.6 k_{\mathrm{B}} T$, and the corresponding interfacial tension $\gamma_{\mathrm{IN}}=0.0053 k_{\mathrm{B}} T / D^{2}$.

An alternative method to obtain the interfacial tension is to perform a finite size scaling analysis. Following Ref. [28], the interfacial tension $\gamma\left(L_{x}\right)$ in a cubic system with edge $L_{x}$, shows a systematic $L_{x}$ dependence that can be written as

$$
\gamma\left(L_{x}\right)=\gamma_{\infty}+a / L_{x}^{2}+b \ln \left(L_{x}\right) / L_{x}^{2},
$$

with $\gamma_{\infty}$ the interfacial tension in the thermodynamic limit (assuming periodic boundary conditions and dimensionality 


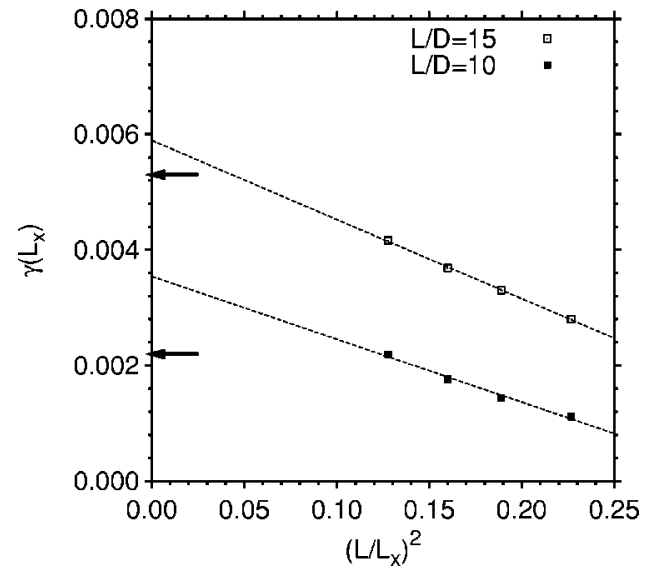

FIG. 7. Finite size extrapolation of the IN interfacial tension of soft spherocylinders with $\epsilon=2$ and rod elongation $L / D=10$ and 15 . Shown is the interfacial tension of the finite system $\gamma\left(L_{x}\right)$ in units of $k_{\mathrm{B}} T / D^{2}$, measured in a cubic system with edge $L_{x}$, as a function of $\left(L / L_{x}\right)^{2}$. Lines are linear fits to the data using Eq. (4) with $b=0$. The upper (lower) arrow indicates the estimate of $\gamma_{\mathrm{IN}}$ obtained using the method of Fig. 6 for $L / D=15(10)$.

$d=3$ ). In general, the constants $a$ and $b$ are not known. However, recent theoretical arguments [36] suggest that in three dimensions, the logarithmic term should vanish, implying $b$ $=0$. To estimate $\gamma_{\infty}$, we used Eq. (3) to measure $\gamma\left(L_{x}\right)$ for a number of different system sizes. We then used Eq. (4) to extrapolate these measurements to the thermodynamic limit, assuming $b=0$. For soft spherocylinders, the results of this procedure are summarized in Fig. 7. Shown is the interfacial tension of the finite system as a function of $\left(L / L_{x}\right)^{2}$. The data seem reasonably well described by Eq. (4), as is indicated by the fits. The corresponding estimates for the interfacial tension are $\gamma_{\mathrm{IN}}=0.0035 k_{\mathrm{B}} T / D^{2}$ and $\gamma_{\mathrm{IN}}=0.0059 k_{\mathrm{B}} T / D^{2}$, for $L / D=10$ and 15 , respectively.

For comparison, the arrows in Fig. 7 mark the interfacial tension as obtained using the previous method of Fig. 6. Clearly, there is some discrepancy. The problem related to the first method is that the system size was not sufficient to completely suppress interface interactions. Moreover, the lateral $L_{x}$ dimension was also rather small, so there may still be finite size effects in this dimension. Hence, we believe the finite size scaling results to be more reliable. The latter estimates are listed in Table I, together with the coexisting phase densities, which effectively summarizes the main results of this work. To our knowledge, this is the first study to report a systematic finite size scaling analysis of the IN interfacial tension in a continuous model (see Ref. [37] for simulation results of a liquid crystal model defined on a lattice). The results of Fig. 7 seem reasonable, but simulations of larger systems are clearly needed, in order to confirm the validity of Eq. (4) in systems of elongated particles. The advantage of the present simulation approach is that the statistical errors are small, and that finite size effects are clearly visible as a result.

\section{DISCUSSION}

In this section, we compare our findings to other work. More precisely, we consider (1) theoretical treatments within the Onsager approximation, (2) theoretical treatments beyond the Onsager approximation, and (3) other simulations. For reasons outlined in the Introduction, we do not compare to experimental data.

It is clear from the phase diagram of Fig. 4 that the Onsager limit is not recovered until very large rod elongation, exceeding at least $L / D=40$. As a result, our estimates for the interfacial tension differ profoundly from Onsager predictions. Typically, $\gamma_{\mathrm{IN}}$ in our simulations is four times lower compared to Onsager estimates. Note that our simulations also show that $\gamma_{\mathrm{IN}}$ increases with $L / D$, toward the Onsager result, so there seems to be qualitative agreement. However, to properly access the Onsager regime, additional simulations for large elongation $L / D$ are required. Unfortunately, as indicated by the scatter in the data of Fig. 4, and also in Ref. [12], such simulations are tremendously complicated. It is questionable if present simulation techniques are sufficiently powerful to extract $\gamma_{\mathrm{IN}}$ with any meaningful accuracy in the Onsager regime.

If we compare to the theory of Ref. [10], which goes beyond the Onsager approximation and should therefore be more accurate for shorter rods, we observe better agreement. For $L / D=10$, the theory predicts $\gamma_{\mathrm{IN}}=0.0877 k_{\mathrm{B}} T /(L+D) D$, which still differs from our result by a factor of approximately 2 . For $L / D=15$, however, a naive interpolation of the data in Ref. [10] yields $\gamma_{\mathrm{IN}} \approx 0.1 k_{\mathrm{B}} T /(L+D) D$, which exceeds our result by only $6 \%$. Note that Ref. [10] considers hard spherocylinders, whereas our work is based on soft spherocylinders. The simulations of Ref. [18] on ellipsoids suggest that the interfacial tension increases, when switching from a hard to a soft potential. The good agreement we observe with Ref. [10] should therefore be treated with some care.

TABLE I. Bulk properties of the coexisting isotropic and nematic phase in a system of soft spherocylinders interacting via Eq. (1) with $\epsilon=2$ and rod elongation $L / D=10$ and 15 . Listed are the reduced density $\rho^{\star}$ and the normalized number density $\rho L D^{2}$ of the isotropic and the nematic phase. Also listed is the interfacial tension $\gamma_{\text {IN }}$ of the IN interface, obtained using finite size scaling, expressed in various units to facilitate the comparison to other work. The error bar in the latter quantity indicates the uncertainty of the fit in Fig. 7.

\begin{tabular}{cccccccc}
\hline \hline \multirow{2}{*}{$L / D$} & \multicolumn{3}{c}{ Isotropic phase } & \multicolumn{2}{c}{ Nematic phase } & \multicolumn{3}{c}{ Interfacial tension $\gamma_{\mathrm{IN}}$} \\
& $\rho^{\star}$ & $\rho L D^{2}$ & $\rho^{\star}$ & $\rho L D^{2}$ & $k_{\mathrm{B}} T / D^{2}$ & $k_{\mathrm{B}} T / L D$ & $k_{\mathrm{B}} T /(L+D) D$ \\
\hline 10 & 0.363 & 0.388 & 0.397 & 0.424 & $0.0035 \pm 0.0003$ & 0.035 & 0.039 \\
15 & 0.244 & 0.267 & 0.280 & 0.307 & $0.0059 \pm 0.0001$ & 0.089 & 0.094 \\
\hline \hline
\end{tabular}


As mentioned in the Introduction, computer simulations of soft ellipsoids with $\kappa=15$ yield interfacial tensions of $\gamma_{\mathrm{IN}}=0.011 \pm 0.004 k_{\mathrm{B}} T / B^{2}$ and $\gamma_{\mathrm{IN}}=0.016 \pm 0.002 k_{\mathrm{B}} T / B^{2}$ $[18,20]$. For $L / D=15$, our result for soft spherocylinders is considerably lower. Obviously, spherocylinders are not ellipsoids, and this may well be the source of the discrepancy. Note also that the shape of the potential used by us is different from that of Refs. $[18,20]$.

In summary, we have performed grand canonical Monte Carlo simulations of the IN transition in a system of soft spherocylinders. By measuring the grand canonical order parameter distribution, the coexistence densities as well as the interfacial tension were obtained. In agreement with theoretical expectations and other simulations, ultralow values for the interfacial tension $\gamma_{\mathrm{IN}}$ are found. Our results confirm that for short rods, the interfacial tension, as well as the coexistence densities, are considerably lower than the Onsager predictions. This demonstrates the need for improved theory to describe the limit of shorter rods, which is required if the connection to experiments is ever to be made. In the future, we hope to extend our simulation method to the case of hard spherocylinders. Note that grand canonical simulations of hard particles are challenging, because the acceptance rate for insertion is typically very low. We are currently investigating different biased sampling techniques in order to improve efficiency. Also, the investigation of the structural properties of the IN interface is in progress.

\section{ACKNOWLEDGMENTS}

We are grateful to the Deutsche Forschungsgemeinschaft (DFG) for support (TR6/A5) and to K. Binder, M. Müller, P. van der Schoot, D. Cleaver, and R. van Roij for stimulating discussions and helpful suggestions. We also thank G. T. Barkema for suggesting some of the numerical optimizations used in this work. T.S. was supported by the Emmy Noether program of the DFG. Allocation of computer time on the JUMP at the Forschungszentrum Juelich is gratefully acknowledged.
[1] L. Onsager, Ann. N.Y. Acad. Sci. 51, 627 (1949).

[2] M. Doi and N. Kuzuu, Appl. Polym. Symp. 41, 65 (1985).

[3] W. E. McMullen, Phys. Rev. A 38, 6384 (1988).

[4] Z. Y. Chen and J. Noolandi, Phys. Rev. A 45, 2389 (1992).

[5] Z. Y. Chen, Phys. Rev. E 47, 3765 (1993).

[6] D. L. Koch and O. G. Harlen, Macromolecules 32, 219 (1999).

[7] K. Shundyak and R. van Roij, J. Phys.: Condens. Matter 13, 4789 (2001).

[8] P. van der Schoot, J. Phys. Chem. B 103, 8804 (1999).

[9] W. Chen and D. G. Gray, Langmuir 18, 663 (2002).

[10] E. Velasco, L. Mederos, and D. E. Sullivan, Phys. Rev. E 66, 021708 (2002).

[11] M. Dijkstra, R. van Roij, and R. Evans, Phys. Rev. E 63, 051703 (2001).

[12] P. Bolhuis and D. Frenkel, J. Chem. Phys. 106, 666 (1997).

[13] M. A. Bates and C. Zannoni, Chem. Phys. Lett. 280, 40 (1997).

[14] M. A. Bates and C. Zannoni, Chem. Phys. Lett. 288, 209 (1998).

[15] M. P. Allen, J. Chem. Phys. 112, 5447 (2000).

[16] M. P. Allen, Chem. Phys. Lett. 331, 513 (2000).

[17] M. S. Al-Barwani and M. P. Allen, Phys. Rev. E 62, 6706 (2000).

[18] A. J. McDonald, M. P. Allen, and F. Schmid, Phys. Rev. E 63, 010701(R) (2000).

[19] A. Z. Panagiotopoulos, Mol. Phys. 61, 813 (1987).

[20] N. Akino, F. Schmid, and M. P. Allen, Phys. Rev. E 63,
041706 (2001).

[21] J. S. Rowlinson and B. Widom, Molecular Theory of Capillarity (Clarendon, Oxford, 1982).

[22] Q. Yan and J. J. de Pablo, J. Chem. Phys. 113, 1276 (2000).

[23] P. Virnau and M. Müller, J. Chem. Phys. 120, 10925 (2004).

[24] J. Potoff and A. Panagiotopoulos, J. Chem. Phys. 112, 6411 (2000).

[25] W. Góźdź, J. Chem. Phys. 119, 3309 (2003).

[26] P. Virnau, M. Müller, L. G. MacDowell, and K. Binder, J. Chem. Phys. 121, 2169 (2004).

[27] R. L. C. Vink and J. Horbach, J. Chem. Phys. 121, 3253 (2004).

[28] K. Binder, Phys. Rev. A 25, 1699 (1982).

[29] D. P. Landau and K. Binder, A Guide to Monte Carlo Simulations in Statistical Physics (Cambridge University Press, Cambridge, 2000).

[30] A. D. Bruce and N. B. Wilding, Phys. Rev. Lett. 68, 193 (1992).

[31] Y. C. Kim, M. E. Fisher, and E. Luijten, Phys. Rev. Lett. 91, 065701 (2003).

[32] S. Wolfsheimer (private communication, 2005).

[33] D. Frenkel and B. Smit, Understanding Molecular Simulation (Academic Press, San Diego, 2001).

[34] M. Müller and N. B. Wilding, Phys. Rev. E 51, 2079 (1995).

[35] N. B. Wilding, Am. J. Phys. 69, 10 (2001).

[36] J. Kaupužs, cond-mat/0501041, 2004.

[37] D. J. Cleaver and M. P. Allen, Mol. Phys. 80, 253 (1993). 